\title{
Detecting planets around very cool dwarfs at near infrared wavelengths with the radial velocity technique
}

\author{
F. Rodler ${ }^{1,2}$, C. del Burgo ${ }^{3}$, S. Witte ${ }^{4}$, Ch. Helling ${ }^{5}$, P. H. Hauschildt ${ }^{4}$, E. L. Martín ${ }^{6}$, C. Álvarez ${ }^{1}$, and R. Deshpande ${ }^{7,8}$ \\ 1 Instituto de Astrofísica de Canarias, C/Vía Láctea s/n, 38205 La Laguna, Spain \\ e-mail: frodler@iac.es \\ 2 Institut de Ciencies de l'Espai (CSIC-IEEC), Campus UAB, Torre C5 - parell - 2a planta, 08193 Bellaterra, Spain \\ 3 UNINOVA-CA3, Campus da Caparica, 2825-149 Caparica, Portugal \\ ${ }^{4}$ Hamburger Sternwarte, Gojenbergsweg 112, 21029 Hamburg, Germany \\ 5 SUPA, School of Physics and Astronomy, University of St. Andrews, North Haugh, St. Andrews KY16 9SS, UK \\ ${ }^{6}$ Centro de Astrobiología (CAB-CSIC), Ctra. Ajalvir km. 4, 28850 Torrejón de Ardoz, Madrid, Spain \\ 7 Center for Exoplanets and Habitable Worlds, The Pennsylvania State University, University Park, PA 16802, USA \\ 8 Department of Astronomy and Astrophysics, The Pennsylvania State University, University Park, PA 16802, USA
}

Received 28 July 2010 / Accepted 12 May 2011

\begin{abstract}
Context. Radial velocity monitoring of very cool dwarfs such as late M- and hot L-dwarfs has become a promising tool in the search for rocky planets as well as follow-up planetary candidates around dwarfs detected by transit surveys. These stars are faint at optical wavelengths, as their spectral flux distribution peaks at near-infrared (NIR) wavelengths. For this reason, it is desirable to measure the radial velocities in this wavelength regime. However, in the NIR very few medium- and high-resolution spectrographs are available at large telescopes. In the near future, high-resolution spectrographs for the NIR will be built, which will allow us to search for rocky planets around cool M-dwarfs and L-dwarfs from radial velocities monitoring.

Aims. We investigate the precision that can be attained in radial velocity measurements of very cool dwarfs in the NIR. The goal is to determine in which atmospheric window of the Earth's atmosphere the highest radial velocity precision can be achieved to help in designing the next generation of NIR high-resolution spectrographs.

Methods. We use stellar atmosphere synthetic models for an M- and an L-dwarf with temperatures of $2200 \mathrm{~K}$ and $1800 \mathrm{~K}$, respectively, and a theoretical spectrum of the Earth's transmission in the spectral range from 0.9 to $2.5 \mu \mathrm{m}$. We simulate a series of Doppler-shifted spectra observed with different resolving powers and signal-to-noise ratios, and for different rotational broadenings of the dwarf. For different combinations of the input parameters, we recover the radial velocity by means of cross-correlation with a high signal-to-noise ratio template and determine the associate uncertainties.

Results. The highest precision in radial velocity measurements for the cool M-dwarf is found in the $Y$ band around $1.0 \mu \mathrm{m}$, while for the L-dwarf it is determined in the $J$ band around $1.25 \mu \mathrm{m}$. We note that synthetic models may lack some faint absorption features or underestimate their abundances. In addition, some instrumental/calibration aspects that are not taken into account in our estimations would increase the uncertainties.
\end{abstract}

Key words. methods: data analysis - techniques: radial velocities - planets and satellites: detection

\section{Introduction}

The search for extrasolar planets has led to about 550 discoveries $^{1}$, mostly detected by means of the radial velocity (RV) technique using high-resolution spectrographs $(R=\lambda / \Delta \lambda \geq 40000)$ at optical wavelengths. Most discoveries are giant gaseous planets (typically hot Jupiters) of short periods (of a few days) around stars of spectral types F and G. To find rocky and low-mass planets, or to follow-up candidates found by means of the transit technique, there are two options: either increase the precision of current spectrographs at optical wavelengths to be less than $0.1 \mathrm{~m} \mathrm{~s}^{-1}$ and search for this kind of planets around F-G type stars, or monitor low-mass stars, such as M- and L-dwarfs, for the following reasons.

Very cool stars such as M-dwarfs are the most abundant type $(\sim 70 \%)$ of stars in the solar neighborhood and the Milky Way in general (Henry et al. 1997). The effective temperatures and masses of M-dwarfs, respectively, are in the range 3700 to

\footnotetext{
1 The Extrasolar Planets Encyclopedia; http://www . exoplanet. eu
}

$2200 \mathrm{~K}$ and 0.5 to 0.07 solar masses for the M0 to M9.5 spectral types. They exhibit prominent absorption features corresponding to strong neutral atoms, $\mathrm{H}_{2} \mathrm{O}, \mathrm{FeH}, \mathrm{VO}, \mathrm{CO}$, and $\mathrm{TiO}$. Owing to the low masses of these objects, the reflex motion of the host star due to the gravitational pull of the extrasolar planet is higher and more easily detectable than for more massive host stars. Since M-dwarfs are very cool stars in comparison with solar-type stars, short period planets would more likely be situated in the habitable zone.

M-dwarfs emit most of their energy around 1.1-1.3 $\mu \mathrm{m}$, in the near-infrared (NIR), while they appear very faint at optical wavelengths. Reiners et al. (2010) investigated the possibilities of searching low-mass planets around M-dwarfs with effective temperatures $3500 \mathrm{~K}$ down to $2600 \mathrm{~K}$. These authors used synthetic spectra from the PHOENIX model atmosphere code (Hauschildt et al. 1999; Allard et al. 2001) for $T_{\text {eff }}=3500 \mathrm{~K}$, $2800 \mathrm{~K}$ and $2600 \mathrm{~K}$ with $\log g=4.75$. As result, they found that the best wavelength range to observe M-dwarfs is in the $Y$ band (i.e. around $1.0 \mu \mathrm{m}$ ). 
First attempts at measuring radial velocity variations among very cool M-dwarfs at NIR wavelengths were done by Martín et al. (2006). Using NIRSPEC (McLean et al. 1998), they achieved a RV precision of around $300 \mathrm{~m} \mathrm{~s}^{-1}$ for the M9.5-dwarf LP944-20. Several research groups have reported high-precision RV measurements taken in the NIR with CRIRES (Käufl et al. 2004), mounted at the UT1/VLT in the ESO Paranal Observatory in Chile. Bean et al. (2010) conducted high-resolution data of 31 M-dwarfs (spectral types M4-M9), and report an RV precision of better than $5 \mathrm{~m} \mathrm{~s}^{-1}$. Figueira et al. (2010) took observations of the planetary candidate TW Hya and achieved a RV precision better than $10 \mathrm{~m} \mathrm{~s}^{-1}$ by adopting telluric lines as a stable reference. However, the exploration of the RVs in the IR is in its infancy, and a revolution is expected in the forthcoming years with the arrival of the new generation of high-resolution nearinfrared spectrographs.

The next cooler class of dwarfs are the L-dwarfs (Kirkpatrick et al. 1999; Martín et al. 1999), which have effective temperatures between $\sim 2100 \mathrm{~K}$ and $1500 \mathrm{~K}$, and their spectral energy distribution peak around $1.3 \mu \mathrm{m}$. Their masses are between $\sim 0.075$ and $0.02 M_{\odot}$.

Charbonneau et al. (2007) presented preliminary first results of a radial velocity survey for exoplanets orbiting L-dwarfs using the PHOENIX spectrograph (Hinkle et al. 2003), which is mounted at the Gemini South telescope. They attained RV precision sufficient to detect Jupiter-mass companions in short-period orbits around L-dwarfs. Using NISPEC at Keck II with a spectra resolving power of $R \sim 25000$, Blake et al. (2010) investigated 59 late M- and L-dwarfs spanning spectral types M8/L0 to L6 over a period of six years. These authors made use of the prominent $\mathrm{CO}$-forest around around $2.3 \mu \mathrm{m}$ in the $K$-band to measure RVs. Although they did not find any planetary companions, they demonstrated that their achieved RV precision of about $50 \mathrm{~m} \mathrm{~s}^{-1}$ for the brighter M-dwarfs and about $200 \mathrm{~m} \mathrm{~s}^{-1}$ for the fainter L-dwarfs is sufficient to detect planets.

In this paper, we investigate the precision that can be attained in RV measurements of a late M-dwarf and an L-dwarf, with an effective temperature of $2200 \mathrm{~K}$ and $1800 \mathrm{~K}$, respectively. Our aim is to define a frame work for the instrumental settings of planned spectrographs of high-resolution in the NIR, which will be devoted to the search for rocky planets around very lowmass stars, and hot brown dwarfs. We focus our study on the four NIR filter bands that lie in high transmission windows of the Earth's atmosphere. In particular, we consider the $Y, J, H$, and $K$ bands, that are located at wavelengths of $1.0-1.1 \mu \mathrm{m}$, $1.17-1.33 \mu \mathrm{m}, 1.5-1.75 \mu \mathrm{m}$, and $2.07-2.35 \mu \mathrm{m}$, respectively.

This paper is organized as follows: Sect. 2 is devoted to a brief discussion of the limiting factors to radial velocity measurements. Section 3 provides a description of the simulations and the data analysis, which is followed by Sect. 4, in which the results are presented. In Sect. 5, our results are discussed.

\section{Limitations to radial velocity measurements of late M-dwarfs in the NIR}

The expected RV precision $\sigma_{\mathrm{RV}}$ can be estimated in general by an equation given in Butler et al. (1996):

$\sigma_{\mathrm{RV}}^{-2}=\sum_{i}\left(\frac{\mathrm{d} I_{i}}{\mathrm{~d} V} S N R_{i}\right)^{2}$,

where $\mathrm{d} I_{i} / \mathrm{d} V$ represent the differential increment in the spectrum (i.e. the first derivative at pixel $i$ ), and $S N R_{i}$ is the signal-to-noise ratio at pixel $i$. Equation (1) means that for a given signal-to-noise ratio $(S N R)$, the RV precision is higher if the star has a large number of deep and narrow absorption lines. Any broadening originating in either the star or the instrument will cause the absorption lines in the stellar spectrum to become both shallower and weaker, leading to a lower precision in the $\mathrm{RV}$ measurements. This broadening is predefined by the spectral resolving power $R=\lambda / \Delta \lambda$ given by the spectrograph and an appropriate sampling of the point spread function at the detector, as well as by stellar rotation (cf. Fig. 4). We briefly discuss the limitations coming from the instrument as well as from the star in Sects. 2.1 and 2.2, respectively.

\subsection{Instrumental limitations}

A crucial part of high-precision RV measurements is the wavelength calibration. This is performed by adopting a stable reference spectrum, which can be a large number of dense absorption lines of a gas sealed in a cell (e.g. iodine gas cell; Butler et al. 2006), which is located in the light path of the target observation, or a dense forest of emission lines of a lamp (e.g. Th-Arlamp; Kerber et al. 2006). These two calibration methods have been successfully carried out in the optical. At NIR wavelengths, however, our knowledge of these calibration methods remains limited, owing to the small number of comparable instrumentation in the NIR.

An advantage of using a gas cell is that the absorption lines of the gas cell are directly superimposed on the stellar spectrum of interest, which allows a direct monitoring of possible changes in the point spread function (PSF) of the instrument. The major disadvantage of adopting a gas cell is that it is hard to find a gas that has strong absorption features in a large wavelength regime, and that gases might absorb a large fraction of the weak stellar light (up to 50\%). The integration times for very cool dwarfs, even with very efficient NIR spectrographs mounted at $8 \mathrm{~m}$ class telescopes are long to ensure a high SNR. Valdivielso et al. (2010) found different mixtures for gas cells providing a dense forest of lines in the NIR in the $H$ and $K$ band. In addition, Mahadevan \& Ge (2009) reported on a mixture that produces a dense absorption spectrum in the $H$ band. Bean et al. (2010) reported that for their CRIRES survey consisting of a search for planets around M-dwarfs, they reach a RV precision of $5 \mathrm{~m} \mathrm{~s}^{-1}$ using a $\mathrm{NH}_{3}$ gas cell in the $K$ band. A similar strategy for wavelength calibration is the use of telluric lines in the NIR as a natural gas cell. In the observations, the telluric lines are superimposed on the stellar spectra, and Seifahrt et al. (2008) showed that these atmospheric lines are stable in terms of velocity in the order of $10 \mathrm{~m} \mathrm{~s}^{-1}$. Figueira et al. (2010) adopted telluric lines as a stable wavelength reference, and showed that these lines allow us to reach RV precisions that are even better than $10 \mathrm{~m} \mathrm{~s}^{-1}$ when accounting for the changing weather conditions at the observatory during the observations.

Special lamps that produce a dense forest of emission lines are used with most of the high-precision and stabilized spectrographs in the optical. For example, the high-precision spectrograph HARPS (Mayor et al. 2003), mounted at the $3.6 \mathrm{~m}$ telescope in La Silla, Chile, allows us to take RV measurements with precisions of less than $1 \mathrm{~m} \mathrm{~s}^{-1}$. The few NIR-spectrographs presently available are also equipped with lamps for wavelength calibration. For example, the intermediate-resolution spectrograph $(R \approx 20000)$ NIRSPEC, mounted on the $10.2 \mathrm{~m}$ Keck II telescope in Hawaii, USA, makes use of arc lamps of different elements, such as $\mathrm{Kr}, \mathrm{Xe}, \mathrm{Ne}$, and $\mathrm{Ar}$, as a stable wavelength reference spectrum. In addition to that, for the 
high-resolution NIR spectrograph CRIRES, mounted on the UT1/VLT at the ESO Paranal Observatory in Chile, a ThAr lamp is used for wavelength calibration. This lamp produces more than 1800 lines in the wavelength range of $\lambda=0.9$ to $2.5 \mu \mathrm{m}$ (Kerber et al. 2008), which is only slightly smaller than in the optical. Controversially for the gas cell, the usage of a lamp does not allow us to monitor changes in the response of the instrument.

\subsection{Limitations from the dwarfs}

Stellar rotation limits the precision that can be attained in RV measurements. In particular, when the rotational broadening function is wider than the PSF, the RV precision is limited to the stellar rotation. For this reason, it is inadvisable to observe rapid rotators with high-resolution when searching for extrasolar planets. Late M-dwarfs are the link between hotter and slowly rotating M-dwarfs and brown dwarfs, which tend to rotate very rapidly. Reiners \& Basri (2010) found that approximately 50\% of their 63 dwarfs of spectral types M7-M9.5 show projected rotational velocities $v \sin i>10 \mathrm{~km} \mathrm{~s}^{-1}$. This fraction of rapid rotators is consistent with a study by Deshpande (2010), who investigated the properties of $36 \mathrm{M}$-dwarfs by using NIRSPEC spectra, and with Jenkins et al. (2009), who determined the projected rotational velocities of $56 \mathrm{M}$-dwarfs.

L-dwarfs have very high rotational velocities. Del Burgo et al. (2009) determined the rotational velocity of brown dwarfs, which had been observed with NIRSPEC spectrograph (McLean et al. 1998) at the Keck II telescope in Hawaii, and found that all of the investigated brown dwarfs have projected rotational velocity larger than $v \sin i \sim 16 \mathrm{~km} \mathrm{~s}^{-1}$.

A well-known problem that hampers attaining highprecision RV measurements is stellar activity, in particular, the presence of stellar spots. These spots lead to line profile distortions in the stellar absorption lines, which can be easily misinterpreted as a RV shift coming from an unseen companion. By observing in the NIR, as opposed to the optical wavelengths, one observes in a domain where the contrast between stellar spots and the stellar disk is lower. Consequently, the semi-amplitude of a RV signal produced by a stellar spot is large at optical wavelengths, and becomes smaller towards infrared wavelengths (e.g. Martín et al. 2006). Reiners et al. (2010) and Barnes et al. (2011) carried out extensive simulations to investigate the effect of a stellar spot on low mass dwarfs on RV measurements at NIR wavelengths. They found that even for small temperature differences between stellar surface and spot, they produce spurious RV-shift with semi-amplitudes larger than $3 \mathrm{~m} \mathrm{~s}^{-1}$. For larger temperature differences, stellar spots can produce spurious shifts with semi-amplitudes larger than $100 \mathrm{~m} \mathrm{~s}^{-1}$. We note that an effective way of distinguishing between spots and real RV-shifts induced by orbiting planets is the analysis of the line bisectors (e.g. Hatzes et al. 1997).

\section{Simulations}

\subsection{Theoretical models for M- and L-dwarfs}

M- and L-dwarfs are very faint in the optical but brighter in the infrared as their spectral energy distribution peaks at around $1.2-1.3 \mu \mathrm{m}$. In the NIR, the spectra of late M-dwarfs show strong alkali lines (K I, Na I, Mn I, Al I), metal hydride bands (FeH, CaH) (Martín et al. 1999), including water, CO, FeH, and VO bands (Mclean et al. 2007; Cushing et al. 2005). In the past few years there has been an emergence of infrared spectroscopic studies which have shed light on their physical characteristics (temperature, mass, radii), e.g. Deshpande (2010), Jackson et al. (2009), and Jenkins et al. (2009).

Early L-dwarfs show a mixture of atomic and molecular bands, the most prominent being the neutral alkali lines ( Na I, $\mathrm{K} \mathrm{I}, \mathrm{Rb}$ I, Cs I, and sometimes Li I), oxide bands TiO and VO, hydride bands $\mathrm{CrH}$ and $\mathrm{FeH}$, and $\mathrm{CaOH}$. Going to cooler L-dwarfs, the ground-state $\mathrm{Na} \mathrm{I}$ and $\mathrm{K}$ I lines grow tremendously in strength; the molecules $\mathrm{MgH}, \mathrm{CaH}, \mathrm{CrH}$, and $\mathrm{FeH}$ become stronger, whereas the oxides $\mathrm{TiO}$ and VO largely disappear. By late- $\mathrm{L}$ and early- $\mathrm{T}, \mathrm{H}_{2} \mathrm{O}$ increases in strength, the neutral alkali lines remain strong, and the hydrides are far less prominent (Kirkpatrick et al. 1999).

Del Burgo et al. (2009, 2010) determined the physical properties (in particular, effective temperature, surface gravity, and rotational broadening) of $\mathrm{M}$ - and T-dwarfs from the comparison of stellar atmosphere synthetic models and $J$-band highresolution (resolving power of $\sim 20000$ ) spectra obtained with the NIRSPEC spectrograph at the Keck II telescope in Hawaii. For their analysis of M-dwarfs with $T_{\text {eff }} \geq 3000 \mathrm{~K}$, they used the general-purpose stellar atmosphere PHOENIX code (Hauschildt \& Baron 1999; Baron et al. 2003). In particular, the version 16 that includes a number of improvements relative to previous versions, such as a complete new equation of state for ions, molecules and condensation, updated opacity databases, and improved line profiles for atomic lines. For $T_{\text {eff }}$ below $3000 \mathrm{~K}$, del Burgo et al. (2009b) used the DriftPHOENIX code to produce theoretical spectra of late $\mathrm{M}$ - and T-dwarfs, which satisfactorily reproduced the observed spectra. That code is a merger of the PHOENIX code and the dust model Drift (Helling et al. 2008a; Witte et al. 2009). The dust grains are composites and yield improved opacities in contrast to the grains in earlier models, and the use of a kinematic, phase-nonequilibrium dust-formation model avoids an overestimated condensation/evaporation (Helling et al. 2008b).

Figure 1 shows the theoretical Drift-PHOENIX spectrum of an M9.5-dwarf as well as for an L-dwarf with an effective temperature of $T_{\text {eff }}=1800 \mathrm{~K}$ in the four atmospheric windows $(Y$, $J, H$, and $K$ bands).

\subsection{Creating the data sets}

To investigate the atmospheric window in which we can achieve the highest RV precision for the late M and L-dwarfs, we create artificial data sets that closely resemble real observations. The free parameters in these data sets were: wavelength band $(Y, J$, $H$, or $K$ band), the stellar rotation $v \sin i$, the spectral resolving power, and the $S N R$ per spectrum.

For the simulations, we adopt two high-resolution DriftPHOENIX models with parameters for the M-dwarf of $T_{\text {eff }}=$ $2200 \mathrm{~K}$ and $\log g=4.5 \mathrm{~cm} \mathrm{~s}^{-2}$, while for the L-dwarf with $T_{\text {eff }}=1800 \mathrm{~K}$ and $\log g=4.5 \mathrm{~cm} \mathrm{~s}^{-2}$ (cf. Fig. 1). The flux of the stellar model is in energy units. To convert the flux of the model into photon units as recorded by the detector, we multiply the flux with the its corresponding wavelength value $\lambda$ throughout the whole spectral range of the model. In the next step, we Doppler-shift this template spectrum by a specified velocity. We account for stellar rotation by convolving the stellar spectrum with a rotational profile, which we computed using the approach described in Gray (2005) for a chosen rotational broadening velocity $v \sin i$ and a limb darkening coefficient of $\epsilon=0.6$.

As the stellar light passes through the Earth's atmosphere, it is partially absorbed by molecules in the atmosphere, such as water and methane. Mathematically, the stellar spectrum is 


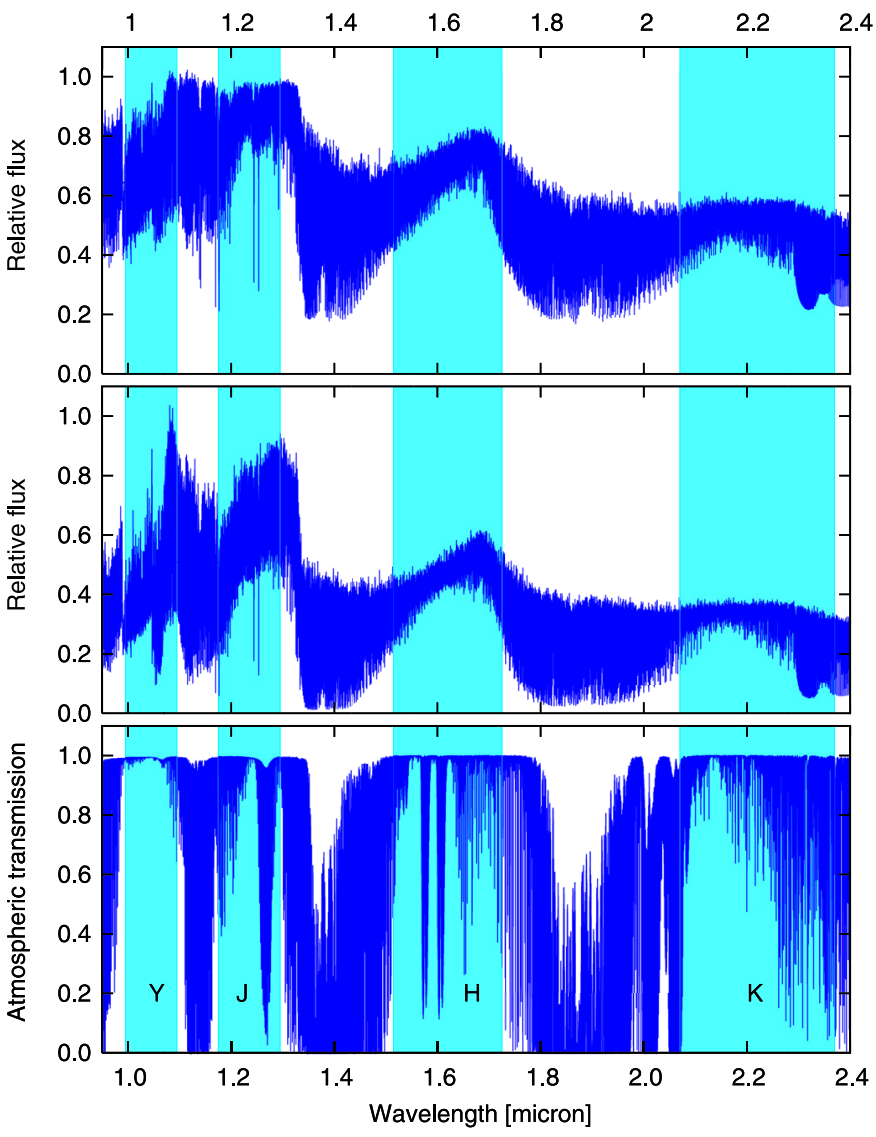

Fig. 1. Bottom: theoretical transmission spectrum of the Earth's atmosphere, calculated for the Observatorio de los Muchachos, La Palma, Spain. The four atmospheric windows $(Y, J, H, K$ band) are indicated. These four transmission bands are not entirely telluric-free: while the $Y$ band shows little contamination, the $J, H$, and $K$ bands show significant telluric contamination. Middle: theoretical spectrum of a hot L-dwarf in the wavelength region between 0.9 and $2.4 \mu \mathrm{m}$, which was generated with the Drift-PHOENIX code for the following parameters: $T_{\text {eff }}=1800 \mathrm{~K}, \log g=4.5 \mathrm{~cm} \mathrm{~s}^{-2}$, and solar metalicity. The positions of the four atmospheric windows $(Y, J, H, K$ band) are indicated. Top: theoretical spectrum of a very cool M-dwarf in the wavelength region between 0.9 and $2.4 \mu \mathrm{m}$, which was generated with the Drift-PHOENIX code for the following parameters: $T_{\text {eff }}=2200 \mathrm{~K}$, $\log g=4.5 \mathrm{~cm} \mathrm{~s}^{-2}$, and solar metalicity. The positions of the four atmospheric windows $(Y, J, H, K$ band) are indicated. While the model spectrum in the $Y$ band shows a forest of deep absorption lines, the spectrum in the $J$ is characterized by shallow features, apart from the strong potassium doublet around $1.25 \mu \mathrm{m}$. Close to the blue and red edge of the $H$ band, deep absorption lines are observed, while the center of this band shows shallow absorption lines. In the $K$ band, the most promising wavelength region is redwards of $2.29 \mu \mathrm{m}$, where a forest of deep CO-absorption lines is present.

multiplied with the transmission spectrum of the Earth's atmosphere. The transmission spectrum was calculated by adopting the line-by-line radiative transfer model (LBLRTM) code, which is based on the FASCODE algorithm (Clough et al. 1992). As a molecular database, we adopt HITRAN (Rothman et al. 2005). We note that we carried out a comparison study between the theoretical transmission spectra and observed mid-resolution $(R \sim 20000)$ spectra, taken with NIRSPEC (McLean et al. 1998) at Keck II in Hawaii, USA, and found that the theoretical spectra reproduce the observations in an excellent way. These results will be presented in a forthcoming paper (Rodler et al., in prep.).
For this paper, we calculate the telluric contamination for the Observatorio de los Muchachos in La Palma, Spain, which is located at $2400 \mathrm{~m}$ above sea level. The strengths of the telluric lines are sensitive to the local elevation in the sky. In our simulations, we assume that we observe our star at low air-masses at an elevation of 65 degrees above horizon. In Fig. 1 (bottom panel), we show the telluric transmission spectrum and mark the four atmospheric windows of interest. This figure depicts that none of these four atmospheric windows is completely telluric-line-free. For this reason, in each band telluric lines appear superimposed on the stellar spectrum. It is obvious that when finally calculating the RVs, these lines needs to be removed from the stellar spectra.

Before the stellar light is recorded at the detector, the light is collected in the telescope and processed by the spectrograph. In this instrument, the stellar spectrum (and the telluric spectrum superimposed on it) is degraded in terms of spectral resolving power and binning on the detector. Mathematically, the stellar spectrum is convolved with the point-spread function (PSF) of the spectrograph. Usually, the PSF of the spectrograph is close to a Gaussian function, but it can be slightly asymmetric (e.g. Endl et al. 2000; Bean et al. 2010). In our simulations, we adopted a Gaussian function as the PSF of the spectrograph. We created data sets for different spectral resolving powers, namely $R=\lambda / \Delta \lambda=20000,40000,60000$ and 80000 , and assumed that the instrument collects the same number of photons at each instrumental setting. The resulting spectra were then interpolated onto a chosen detector grid, with a two-pixel sampling per resolution element, where the spectral resolving power was kept constant across the full spectral range.

In the final step, we add Poisson noise to the data according to the certain $S N R$ in the stellar continuum at a selected wavelength $\lambda$. We assume that the detector has the same sensitivity throughout the observed wavelength region, and we take into account the spectral flux distribution of the dwarf. To this end, we simply scale the spectrum so that the spectral flux distribution information is conserved and the maximum flux is the squared value of the desired $S N R$. The actual $S N R$ per spectral pixel is then determined as the square root of the flux at that pixel. When finally adding Photon noise to the data, this approach ensures that regions of telluric lines as well as stellar absorption lines have a lower $S N R$, respectively.

\subsection{Analysis of the data sets}

After having created our "observations", we apply the same analysis we would follow when dealing with truly observed spectra. For the removal of the telluric contamination in our data analysis, we investigate two cases: (a) the first one represents the complete and perfect removal of the telluric lines to determine the highest RV precision possible. The only indicator for the removed telluric lines is a reduced $S N R$ in those regions. In the more realistic approach (b), we chose a similar approach as presented in Reiners et al. (2009) and mask out those telluric lines with absorption depths $\geq 3 \%$ at a spectral resolving power of $R=80000$. In addition, owing to the close vicinity $\left( \pm 30 \mathrm{~km} \mathrm{~s}^{-1}\right)$ of these lines, we discard them from further analysis to account for the velocity shifts produced by the barycentric motion of the Earth.

To avoid the effects of the dispersion (e.g. the spectral lines becoming broader towards longer wavelengths), we convert the dispersion axis from wavelength $\lambda$ to velocity $v$. 
F. Rodler et al.: Detecting planets around very cool dwarfs at near infrared wavelengths with the radial velocity technique

To this end, we apply the equation

$v=\frac{c}{\lambda} \mathrm{d} \lambda=c \ln \lambda$,

where $c$ is the speed of light in a given unit, which is then the unit of the velocity grid.

In the next step, we subdivide the spectrum into equidistant pixel chunks each of size 200 pixel. We assign each chunk a weight $w_{i}$ according to the average SNR of the "observation". For each wavelength chunk, we cross-correlate the "observation" with a template, which is nothing but a high-SNR reference spectrum of the "observation". We then calculate the relative RV with respect to the template. These chunks that show RV values that are clearly outliers are discarded from further analysis. Finally, we determine the error in the RV-measurement as:

$\sigma_{\mathrm{RV}}=\frac{\mathrm{rms} w_{i}}{\sqrt{\sum w_{i}}}$

where $w_{i}$ is the weight of the unrejected chunk $i$.

\section{Results}

\subsection{M9.5-dwarf}

We analyzed data sets of the M9.5-dwarf in the four different atmospheric windows and four different spectral resolving powers, which had the following configuration: a stellar rotation of $v \sin i=5 \mathrm{~km} \mathrm{~s}^{-1}$, and a $S N R=90$ in stellar continuum at the peak of the spectral energy distribution of the dwarf at a spectral resolving power of $R=80000$ to account for the intrinsic faintness of these class of objects. The corresponding $S N R$ values in the other wavelength bands and spectral resolving powers are given in Table 1.

Figure 2 shows the analysis for the case in which the telluric lines were completely and perfectly removed (Case A), while Fig. 3 depicts the analysis where telluric regions are discarded from analysis (Case B). The highest RV precision is attained in the $Y$ bandwidth with $v=3.4 \mathrm{~m} \mathrm{~s}^{-1}$ and $v=3.9 \mathrm{~m} \mathrm{~s}^{-1}$ for a spectral resolving power of $R=80000$, respectively, for the Cases A and B. In our simulations, we show the prefect scenario, i.e. perfect wavelength solution and a complete wavelength coverage throughout the transmission bands without inter-order gaps.

Figure 4 shows the results of our studies of how the RV precision depends on the stellar rotation $v \sin i$ and the spectral resolving power. We carried out the simulations in the $Y$ band with the SNR values in the stellar continua given in Table 1. In this figure, we see that for slowly rotating stars $\left(v \sin i<10 \mathrm{~km} \mathrm{~s}^{-1}\right)$, observing at higher resolution allows us to take RV measurements with a smaller RV error. However, for rapidly rotators, there is hardly no improvement in terms of achievable RV precision between the spectral resolving powers of $R=60000$ and 80000 , since the width of the rotational broadening function is roughly the same as the width of the PSF of the spectrograph.

We furthermore investigated the kind of planets that could be discovered with the attained RV precisions. We have to remind the reader that in our simulations, we accounted neither for wavelength calibration errors nor other instrumental issues, which could introduce noise to the data and lower the RV precisions. Consequently, the following numbers denote the best case scenario and the RV precision might be lower in reality.

We determined the minimum mass of the planet by employing the equation

$m_{\mathrm{p}, \min }=K_{\star} m_{\star}^{1 / 3}\left(\frac{P}{2 \pi \mathrm{G}}\right)^{2 / 3}$,
Table 1. Radial velocity precisions of the dwarfs that can be attained for spectral resolving powers $R$ and $S N R$ in the stellar continua.

\begin{tabular}{lcccccccc}
\hline \hline$R$ & \multicolumn{4}{c}{ SNR } & \multicolumn{4}{c}{ RV precision $\left(\mathrm{m} \mathrm{s}^{-1}\right)$} \\
& $Y$ & $J$ & $H$ & $K$ & $Y$ & $J$ & $H$ & $K$ \\
\hline \multicolumn{4}{c}{ M9.5V-dwarf $\left(T_{\text {eff }}=2200 \mathrm{~K}\right)+$ Case A } \\
20000 & 139 & 180 & 171 & 152 & 22.2 & 25.5 & 22.8 & 27.9 \\
40000 & 98 & 127 & 121 & 108 & 6.9 & 8.7 & 7.8 & 10.8 \\
60000 & 80 & 104 & 99 & 88 & 4.2 & 5.7 & 5.1 & 3.8 \\
80000 & 70 & 90 & 85 & 76 & 3.3 & 4.0 & 3.8 & 5.1 \\
\hline \multicolumn{4}{c}{ M9.5V-dwarf $\left(T_{\text {eff }}=2200 \mathrm{~K}\right)+$ Case B } \\
20000 & 139 & 180 & 171 & 152 & 24.2 & 29.7 & 29.1 & 39.3 \\
40000 & 98 & 127 & 121 & 108 & 8.7 & 12.2 & 11.9 & 17.3 \\
60000 & 80 & 104 & 99 & 88 & 5.4 & 7.1 & 6.8 & 10.7 \\
80000 & 70 & 90 & 85 & 76 & 3.8 & 5.2 & 5.1 & 7.9 \\
\hline \multicolumn{1}{c}{ L-dwarf $\left(T_{\text {eff }}=1800 \mathrm{~K}\right)+$ Case A } \\
20000 & 68 & 90 & 88 & 84 & 83.2 & 73.8 & 92.5 & 92.5 \\
40000 & 47 & 64 & 63 & 59 & 60.6 & 56.8 & 66.9 & 66.9 \\
60000 & 38 & 52 & 52 & 50 & 49.0 & 45.1 & 53.5 & 53.5 \\
80000 & 33 & 45 & 44 & 42 & 42.3 & 37.5 & 57.0 & 57.0 \\
\hline \multicolumn{1}{c}{ L-dwarf $\left(T_{\text {eff }}=1800 \mathrm{~K}\right)+$ Case B } \\
20000 & 68 & 90 & 88 & 84 & 87.6 & 77.0 & 109.2 & 158.3 \\
40000 & 47 & 64 & 63 & 59 & 65.9 & 61.8 & 84.2 & 114.5 \\
60000 & 38 & 52 & 52 & 50 & 55.3 & 50.9 & 70.0 & 95.6 \\
80000 & 33 & 45 & 44 & 42 & 47.3 & 41.4 & 60.7 & 79.8 \\
\hline
\end{tabular}

Notes. Case A shows the data analysis case of a complete and perfect removal telluric contamination, while Case B lists the RV precisions for the data analysis, where the telluric lines of a absorption depth of $\geq 3 \%$ were masked out.

where $K_{\star}$ is the RV semi-amplitude of the reflex motion of the star, $m_{\star}$ is the stellar mass, $P$ the orbital period of the planet, and $\mathrm{G}$ the gravitational constant. We considered only the case of a circular orbit. We set the mass of the very cool M-dwarf to $m_{\star}=0.075 M_{\odot}$, and calculated the minimum masses of the planet with respect to the orbital period $P$ and $K_{\star}$. Figure 7 shows the results we determined for different spectral resolving powers in the optimal wavelength band ( $Y$ band). For example, if we obtain a spectrum of an M9.5-dwarf with a rotational broadening of $v \sin i=5 \mathrm{~km} \mathrm{~s}^{-1}$, in the $Y$ band with a $S N R$ of 80 in the stellar continuum at a spectral resolving power of $R \geq 60000$, we are able to detect short period planets with minimum masses $m_{\mathrm{p}, \min } \sim 2 M_{\oplus}$.

\subsection{L-dwarf}

We analyzed data sets of the L-dwarf with a temperature of $1800 \mathrm{~K}$ in the four different atmospheric windows and four different spectral resolving powers, which had the following configuration: to account for the rapid rotation of brown dwarfs, we set the stellar rotation to $v \sin i=20 \mathrm{~km} \mathrm{~s}^{-1}$. In addition, to account for the relative faintness of hot L-dwarfs by adopting a $S N R=45$ in the stellar continuum at the peak of the spectral energy distribution of the L-dwarf at a spectral resolving power of $R=80000$. The corresponding $S N R$ values in the other wavelength bands and spectral resolving powers are given in Table 1.

Figure 5 shows the results of Case A where the telluric lines were completely and perfectly removed from the spectra, while in Fig. 6 we show the results of Case B where the regions of telluric contamination were masked out and discarded from further analysis. As a result, for both cases we find that the highest $\mathrm{RV}$ precision for the L-dwarf is achieved in the $J$. The RV precisions for the different wavelength regions and spectral resolving powers are given in Table 1. 


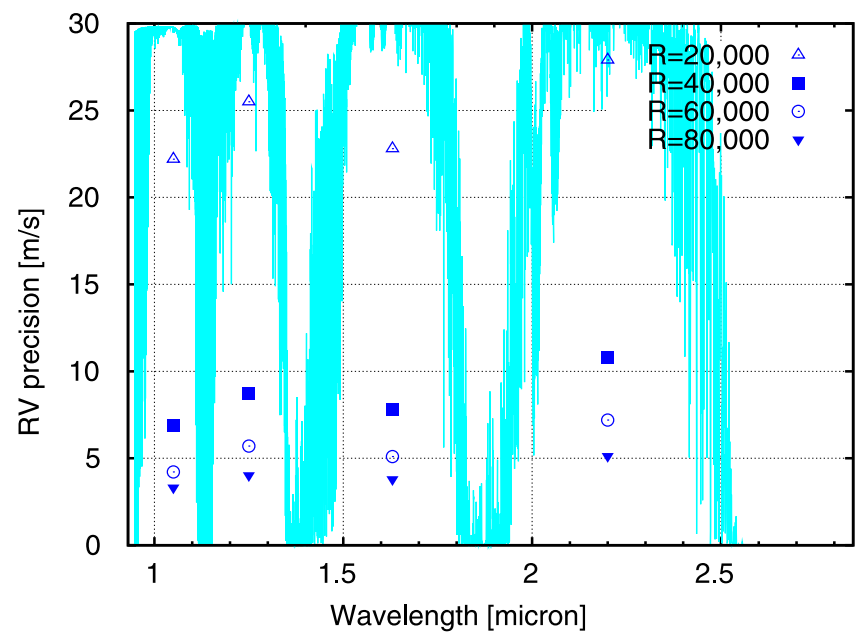

Fig. 2. Expected RV precisions of an M9.5-dwarf with a temperature of $2200 \mathrm{~K}$ and a projected rotational velocity of $v \sin i=5 \mathrm{~km} \mathrm{~s}^{-1}$ in the different NIR wavelength bands $(Y, J, H$ and $K)$ for different spectral resolving powers. For these simulations, we accounted for the spectral flux distribution of the M9.5-dwarf, and assumed a homogeneously sensitive detector throughout the NIR. The gray spectrum in the background represents the transmission of the Earth's atmosphere (the value 30 in the $y$-axis corresponds to $100 \%$ transmission). The SNR in the brightest regions in the stellar continuum of the M-dwarf was set to be 90 for a spectral resolving power of $R=80000$. The $S N R$ values and the RV precision values for the different wavelength bands and spectral resolving powers are given in Table 1 . Although the spectral flux distribution of such a cool dwarf peaks around $1.2 \mu \mathrm{m}$, the highest radial velocity precision is attained in the $Y$ band.

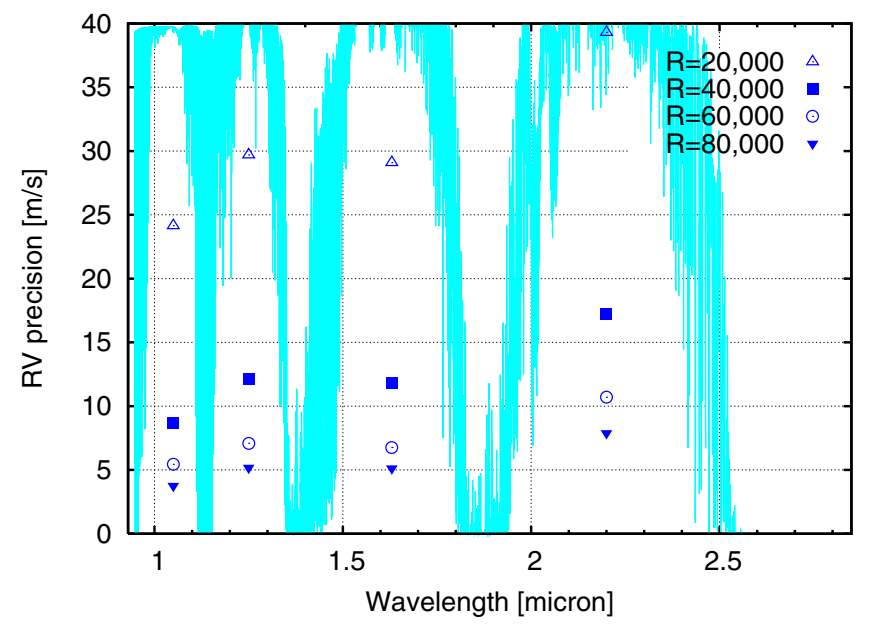

Fig. 3. Same as Fig. 2, but for Case B, where we masked out regions of heavy telluric contamination (i.e. $3 \%$ or more of telluric absorption at $R=80000$ ) in our analysis. The gray spectrum in the background represents the transmission of the Earth's atmosphere (the value 40 in the $y$-axis corresponds to $100 \%$ transmission). The highest radial velocity precision is attained in the $Y$ band.

Adopting Eq. (4) and the RV precisions we had previously determined for the different spectral resolving powers in the $Y$ band, we calculated the minimum masses of possible planetary companions to the L-dwarf with respect to the orbital period. In Fig. 7 we show that hot Neptunes could be detected with minimum masses of $m_{\mathrm{p}, \min }>20 M_{\oplus}$ and periods $\leq 5$ days, when observations are taken at a spectral resolving power of $R \geq 60000$, and a conservative value for the mass of the L-dwarf with $m_{\star}=0.07 M_{\odot}$ is assumed.

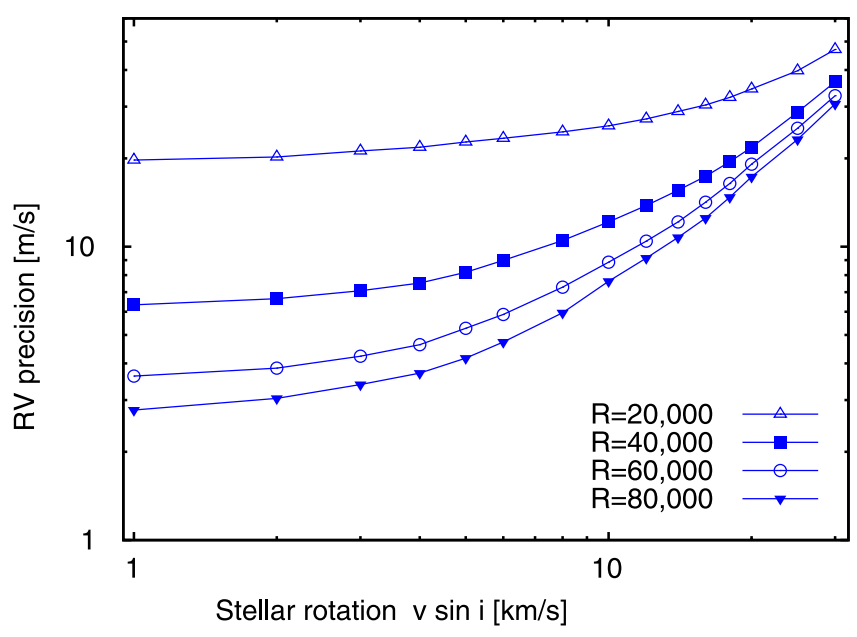

Fig. 4. Achievable radial velocity of the very cool M-dwarf as a function of the stellar rotation $v \sin i$ and the spectral resolving powers. For the simulations, we created data sets for the $Y$ band with the average $S N R$ given in Table 1 in the stellar continuum. The free parameters for the simulation were the stellar rotation and the spectral resolving power.

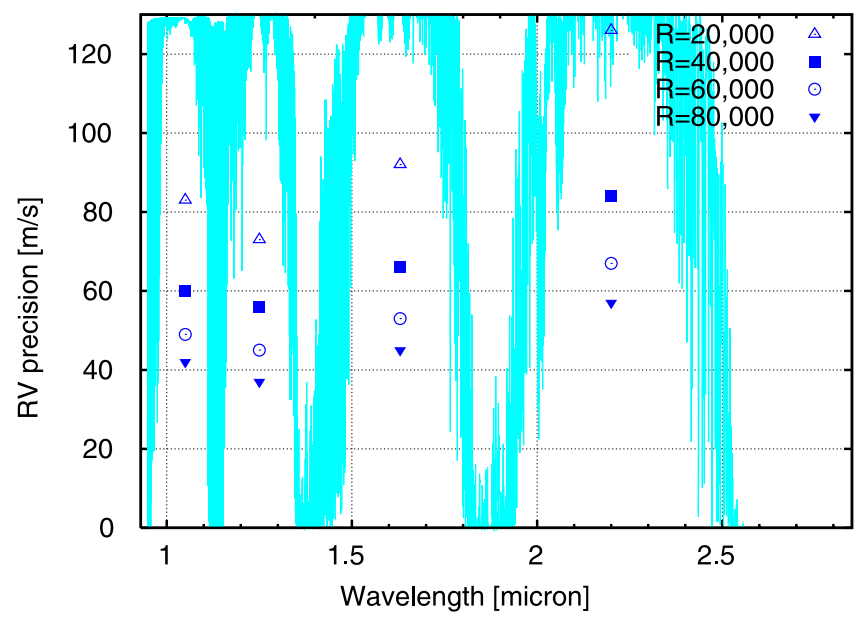

Fig. 5. Expected RV precisions of hot brown dwarf with a temperature of $1800 \mathrm{~K}$ and a projected rotational velocity of $v \sin i=20 \mathrm{~km} \mathrm{~s}^{-1}$ in the different NIR wavelength bands $(Y, J, H$, and $K)$ for different spectral resolving powers. For these simulations, we accounted for the spectral flux distribution of the brown dwarf, and assumed a homogeneously sensitive detector throughout the NIR. The SNR in the brightest regions in the continuum of the brown dwarf was set to be 45 for a spectral resolving power of $R=80000$. The $S N R$ values and the RV precision values for the different wavelength bands and spectral resolving powers are given in Table 1. The gray spectrum in the background represents the transmission of the Earth's atmosphere (the value 130 in the $y$-axis corresponds to $100 \%$ transmission). The highest radial velocity precision is attained in the $J$ band.

\section{Summary and discussion}

We have investigated which atmospheric window in the NIR (i.e. $Y, J, H$, and $K$ band) is best-suited for the search for rocky planets around a very cool M-dwarf and L-dwarf with a temperature of $T_{\text {eff }}=2200 \mathrm{~K}$ and $1800 \mathrm{~K}$, respectively. We carried out the simulation for two different cases of the removal of the telluric contamination in our analysis: Case A was an analysis with a complete and perfect removal of telluric lines from the spectra to asses which RV precision would be possible in the best case for different instrumental settings, while in the more realistic Case B regions at and around telluric absorption lines of $\geq 3 \%$ 
F. Rodler et al.: Detecting planets around very cool dwarfs at near infrared wavelengths with the radial velocity technique

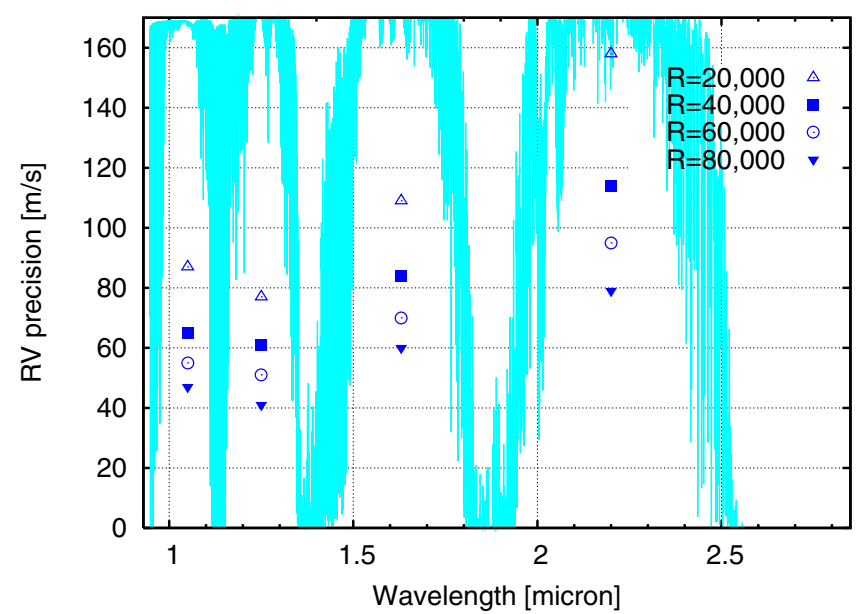

Fig. 6. Same as Fig. 5, but for Case B, where we masked out regions of heavy telluric contamination (i.e. $3 \%$ or more of telluric absorption at $R=80000)$ in our analysis. The gray spectrum in the background represents the transmission of the Earth's atmosphere (the value 170 in the $y$-axis corresponds to $100 \%$ transmission). The highest radial velocity precision is attained in the $J$ band.

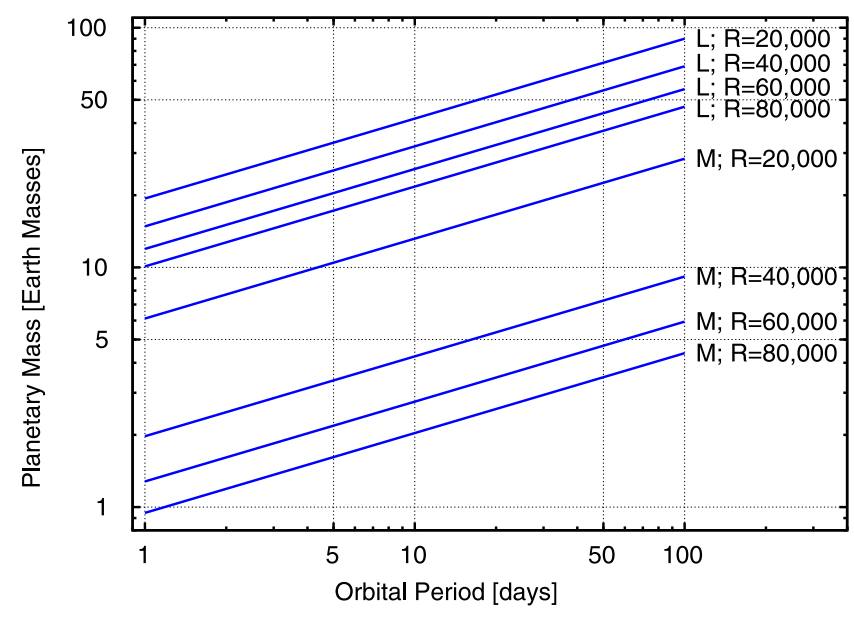

Fig. 7. Minimum masses of the planetary companions with respect to the orbital periods and the RV precisions, which are a function of the spectral resolving power $R$. For the late M-dwarf (in the plot marked with "M") we assumed a mass of $0.075 M_{\odot}$ and a projected rotational velocity of $v \sin i=5 \mathrm{~km} \mathrm{~s}^{-1}$. We calculated the masses by adopting Eq. (4) and the RV precision we had determined for different spectral resolving powers $(R=20000,40000,60000$ and 80000$)$ in the $Y$ band with a $S N R$ of 80 in the spectral continuum. We demonstrate that planetary companions with short periods and slightly higher masses than the Earth can be detected when taking observations at high spectral resolving powers. For the L-dwarf (marked with "L") with a temperature of $1800 \mathrm{~K}$, we assumed a mass of $0.07 M_{\odot}$ and a projected rotational velocity of $v \sin i=20 \mathrm{~km} \mathrm{~s}^{-1}$. As result, we find that hot Neptunes can be detected when observations are taken in the $J$ band, with a spectral resolving power of $R \geq 60000$ and a $S N R$ of 45 in the spectral continuum.

depth are masked out and discarded from the RV determination process.

We note that the RV precisions presented in this paper are estimates, since our simulations are based on assumptions. We have used theoretical PHOENIX-Drift model spectra to describe the dwarfs. These synthetic models may lack some faint absorption features or underestimate their abundances. Conversely, some instrumental/calibration aspects, such as the errors coming from the wavelength calibration or noise coming from the detector (e.g. read-out noise, sky background variations, and thermal noise), as well as possible stellar activity are not taken into account in our estimations, which would all increase the uncertainties and lower the RV precision.

For the M9.5 dwarf, we found that for both cases of treating the telluric contamination that the highest RV precision can be achieved in the $Y$ band. Our results support the conclusions drawn by Reiners et al. (2010) for their choice of hotter M-dwarfs ( $T_{\text {eff }}=3500 \mathrm{~K}, 2800 \mathrm{~K}$ and $\left.2600 \mathrm{~K}\right)$. We have demonstrated that planetary companions with short periods and slightly higher masses than the Earth can already be detected when taking observations in the $Y$ band at spectral resolving powers of $R \geq 60000$. Furthermore, we have studied the dependence of the stellar rotation and the spectral resolving power on the achievable RV precision. For all the rotational velocities the highest spectral resolving power of 80000 yields the smallest errors in the RV measurements. However, for rapid rotators $\left(v \sin i \geq 10 \mathrm{~km} \mathrm{~s}^{-1}\right)$, we see hardly any difference in RV precision between a spectral resolving power of 60000 and 80000 .

For the L-dwarf with a temperature of $1800 \mathrm{~K}$, the highest $\mathrm{RV}$ precision in both cases of treating the telluric contamination is attained in the $J$ band. Since these objects are intrinsically very faint and tend to rotate rapidly, the achievable RV precisions are about a magnitude higher than those for late M-dwarfs. Nevertheless, we have demonstrated that at least hot Neptunes could be discovered around L-dwarfs when taking observations at spectral resolving powers of $R \geq 60000$.

We furthermore compared the RV precisions for both cases of telluric line treatment. In the $Y$ band, the difference in RV precision between Case A and B is relatively small, due to the low contamination in this wavelength band. In the $J$ and $H$ bands, we found relatively moderate telluric contamination, which nevertheless affects the RV determination process. However, in the $K$ band, heavy telluric contamination throughout the whole wavelength band severely affects the RV precision (cf. Table 1 and Fig. 1).

Our studies bring out the need for high-resolution crossdispersed spectrographs to search for rocky planets and/or to follow-up transit candidates around cool M-dwarfs and L-dwarfs. To avoid problems with the wavelength calibration and ensure the stability required, we suggest that such spectrographs should only have one observing mode as well as allow us to take Th-Ar exposures simultaneously with the target observations. The spectrograph should simultaneously cover the $Y$, $J$, and $H$ band, but avoid the $K$ band. Extending the spectrograph to the latter atmospheric window would require severe cooling and different optical parts (e.g. different fibers which have a high optical throughput in the $K$ band). Since roughly half of the cool M-dwarfs as well as most of the L-dwarfs are rapid rotators, a spectral resolving power of $R \sim 60000$ is sufficient to achieve high RV precisions. A practical advantage of a lower resolution with respect to the spectral resolving power of $R=80000$ would be that inter-order gaps between the spectral orders would remain relatively small, and consequently the wavelength coverage would be higher.

The results of our simulations led to a re-design of the high-resolution part of the NIRINTS spectrograph (formerly NAHUAL; Martín et al. 2005), to be mounted on the GTC at the Observatorio de los Muchachos in La Palma, Spain. This spectrograph was designed to have a spectral resolving power of $R \sim 61000$ and to cover the entire $Y, J$ and $H$ bands. Its main science goals were to detect and follow-up rocky planets around cool M-dwarfs and L-dwarfs. 
Further projects to build high-resolution spectrographs covering one or more atmospheric windows in the NIR have been launched. For example, the project CARMENES (Quirrenbach et al. 2010), with a proposed arm in the NIR having a spectral resolving power of $R \sim 85000$, covering the $Y$ and $J$ bands, should be mounted at the $3.5 \mathrm{~m}$ telescope of the Calar Alto Observatory in Spain. The science case for this spectrograph is to monitor (massive) M-dwarfs. The Habitable Zone Planet Finder (HZPF) is a proposed instrument for the $10 \mathrm{~m}$ class Hobby Eberly telescope at the McDonald observatory in Texas, USA, which will provide a spectral resolving power of $R \sim 50000$ and cover the $Y, J$, and parts of the $H$ bands (Mahadevan et al. 2010). This instrument will be capable of discovering low mass planets around M dwarfs.

Acknowledgements. This work has been supported by the Spanish Ministerio de Eduación y Ciencia through grant AYA2007-67458. F.R. is very grateful to Andreas Seifahrt, who helped to install LBLRTM and HITRAN to compute transmission spectra of the Earth's atmosphere. Furthermore, we thank the anonymous referee for very important suggestions.

\section{References}

Allard, F., Hauschildt, P. H., Alexander, D., et al. 2001, ApJ, 556, 357

Baron, E., Hauschildt, P. H., Allard, F., et al. 2003, IAU Symp., 210, 19

Barnes, J. R., Jeffers, S. V., \& Jones, H. R. A. 2011, MNRAS, 412, 1599

Bean, J. L., Seifahrt, A., Hartman, H., et al. 2010, ApJ, 713, 410

Butler, R. P., Marcy, G. W., Williams, E., et al. 1996, PASP, 108, 500

Charbonneau, D., Blake, C. H., White, R. J., \& Marley, M. S. 2007, Bull. AAS, 38,145

Clough, S. A., Iacono, M. J., \& Moncet, J.-L. 1992, J. Geophys. Res., 97, 15761 Cushing, M. C., Rayner, J. T., \& Vacca, W. D. 2005, ApJ, 623, 1115

del Burgo, C., Martín, E. L., Zapatero Osorio, M. R., \& Hauschildt, P. 2009, A\&A, 501, 1059

del Burgo, C., Deshpande, R., Martín, E. L., et al. 2010, Proc. Conf., New Technologies for Probing the Diversity of Brown Dwarfs and Exoplanets, in Shanghai
Deshpande, R. 2010, Ph.D.-Thesis, University of Central Florida Endl, M., Kürster, M., \& Els, S. 2000, A\&A, 362, 585

Figueira, P., Pepe, F., Melo, C. H. F., et al. 2010, A\&A, 511, 55

Gray, D. F. 2005, Stellar Photospheres, Third Edition (Cambridge University Press)

Hatzes, A. P., Cochran, W. D., \& Johns-Krull, Ch. M. 1997, ApJ, 478, 374

Hauschildt, P. E., \& Baron, E. 1999, JCoAM, 109, 41

Hauschildt, P. H., Allard, F., Ferguson, J., et al. 1999, ApJ, 525, 871

Helling, Ch., Woitke, P.-R., \& Thi, F. 2008a, A\&A, 485, 547

Helling, Ch., Dehn, M., Woitke, P., \& Hauschildt, P. H. 2008b, ApJ, 675, 105

Henry, T., Ianna, P., Kirkpatrick, D., et al. 1997, ApJ, 114, 388

Hinkle, K. H., Blum, R., Joyce, R. R., et al. 2003, SPIE, 4834, 353

Jackson, R. J., Jeffries, R. D., \& Maxted, P. F. L. 2009, MNRAS, 399, 89

Jenkins, J. S., Ramsey, L. W., Jones, H. R. A., et al. 2009, ApJ, 704, 975

Käufl, H.-U., Ballester, P., Biereichel, P., et al. 2004, in SPIE Conf. Ser. 5492, ed. A. F. M. Moorwood, \& M. Iye, 1218

Kerber, F., Nave, G., \& Sansonetti, C. J. 2006, SPIE, 6269, 83

Kerber, F., Nave, G., \& Sansonetti, C. J. 2008, ApJS, 178, 374

Kirkpatrick, J. D., Reid, I. N., Liebert, J., et al. 1999, ApJ, 519, 802

Mahadevan, S., \& Ge, J. 2009, ApJ, 692, 1590

Mahadevan, S., Ramsey, L., Wright, J., et al. 2010, in Ground-based and Airborne Instrumentation for Astronomy III, ed. I. S. McLean, S. K. Ramsay, \& H. Takami, Proc. SPIE, 7735, 77356X [arXiv: 1007. 3235]

Martín, E. L., Delfosse, X., Basri, G., et al. 1999, AJ, 118, 2466

Martín, E. L., Guenther, E., Barrado y Navascués, D., et al. 2005, AN, 326, 1015

Martín, E. L., Guenther, E., Zapatero Osorio, M. R., Bouy, H., \& Wainscoat, R. J. 2006, ApJ, 644, L75

Mayor, M., Pepe, F., Queloz, D., et al. 2003, The ESO Messenger, 114, 20

McLean, I. S., Becklin, E. E., Bendiksen, O., et al. 1998, SPIE, 3354, 566

McLean, I. S., Prato, L., McGovern, M. R., et al. 2007, ApJ, 658, 1217

Reiners, A., \& Basri, G. 2010, ApJ, 710, 924

Reiners, A., Bean, J. L., Huber, K. F., et al. 2010, ApJ, 710, 432

Rothman, L. S., Jacquemart, D., Barbe, A., et al. 2005, JQSRT, 96, 139

Seifahrt, A., \& Käufl, H. U. 2008, A\&A, 491, 929

Quirrenbach, A., Amado, P. J., Mandel, H., et al. 2010, SPIE, 7735, 37

Valdivielso, L., Epearza, P., Martín, E., et al. 2010, ApJ, 715, 1366

Witte, S., Helling, Ch., \& Hauschildt, P. H. 2009, A\&A, 506, 1367 